Proyecciones Journal of Mathematics

Vol. 33, No 2, pp. 189-204, June 2014.

Universidad Católica del Norte

Antofagasta - Chile

\title{
A class of multivalent functions defined by generalized Ruscheweyh derivatives involving a general fractional derivative operator
}

\author{
Hari Singh Parihar \\ Central University of Rajasthan, India \\ and \\ Ritu Agarwal \\ Malaviya National Institute of Technology, India \\ Received: March 2013. Accepted : September 2013
}

\begin{abstract}
The main aim of the present paper is to obtain a new class of multivalent functions which is defined by making use of the generalized Ruscheweyh derivatives involving a general fractional derivative operator. We study the region of starlikeness and convexity of the class $\Omega_{p}(\alpha, \beta, \gamma)$. Also we apply the Fractional calculus techniques to obtain the applications of the class $\Omega_{p}(\alpha, \beta, \gamma)$. Finally, the familiar concept of $\delta$-neighborhoods of p-valent functions for above mentioned class are employed.
\end{abstract}

Subject class (2010) : Primary 26A33, Secondary 30C45.

Keywords : Starlike function, p-valent function, Convolution, Generalized fractional derivative operator, Generalized Ruscheweyh derivatives 


\section{Introduction}

Let $A$ denote the class of functions that are analytic in the open unit disk $U=\{z \in C:|z|<1\}$ and let $A_{p}$ be the subclass of $A$ consisting of the functions $f$ of the form

$$
f(z)=z^{p}-\sum_{k=n+p}^{\infty} a_{k} z^{k}, \quad(n \in N)
$$

where $\mathrm{p}$ is some positive integer and $f$ is analytic and $\mathrm{p}$-valent in $\mathrm{U}$.

The generalized fractional derivative operator of order $\lambda$, introduced by Srivastava and Saxena [9], [10], is defined as

$$
J_{0, z}^{\lambda, \mu, \nu} f(z)=\left\{\begin{array}{l}
\frac{1}{\Gamma(1-\lambda)} \frac{d}{d z}\left\{z^{\lambda-\mu} \int_{0}^{z}(z-\zeta)^{-\lambda}\right. \\
\left.\cdot{ }_{2} F_{1}\left(\mu-\lambda, 1-\nu ; 1-\lambda ; 1-\frac{\zeta}{z}\right) f(\zeta) d \zeta\right\}, \\
\quad(0 \leq \lambda<1) \\
\frac{d^{n}}{d z^{n}} J_{0, z}^{\lambda-n, \mu, \nu} f(z), \quad(n \leq \lambda<n+1, n \in N)
\end{array}\right.
$$

where $f$ is an analytic function in a simply connected region of the zplane containing the origin, and the multiplicity of $(z-\zeta)^{-\lambda}$ is removed by requiring $\log (z-\zeta)$ to be real when $z-\zeta>0$, provided further that,

$$
f(z)=O\left(|z|^{k}\right), \quad(z \rightarrow 0)
$$

In terms of gamma function, we have

$$
\begin{aligned}
J_{0, z}^{\lambda, \mu, \nu} z^{\rho}= & \frac{\Gamma(\rho+1) \Gamma(\rho-\mu+\nu+2)}{\Gamma(\rho-\mu+1) \Gamma(\rho-\lambda+\nu+2)} z^{\rho-\mu}, \\
& (0 \leq \lambda<1, \rho>\max \{0, \mu-\nu-1\}-1)
\end{aligned}
$$

It follows at once from the above definition that

$$
J_{0, z}^{\lambda, \lambda, \nu} f(z)=D_{z}^{\lambda} f(z)=\frac{1}{\Gamma \lambda} \int_{0}^{z} \frac{f(t)}{(z-t)^{1-\delta}} d t, \quad(0 \leq \lambda<1) .
$$


where $D_{z}^{\lambda} f(z)$ is the fractional derivative operator of order $\lambda$. Furthermore, in terms of gamma function, we have

$$
D_{z}^{\lambda} z^{\rho}=\frac{\Gamma(\rho+1)}{\Gamma(\rho-\delta+1)} z^{\rho-\lambda}, \quad(0 \leq \lambda)
$$

Similary, the fractional integral opearator of order $\lambda$ is

$$
D_{z}^{-\lambda} f(z)=\frac{1}{\Gamma(1-\lambda)} \frac{d}{d z} \int_{0}^{z} \frac{f(t)}{(z-t)^{\delta}} d t
$$

where $f$ is an analytic function in a simply connected region of the $\mathrm{z}$ plane containing the origin, and the multiplicity of $(z-t)^{-\lambda}$ is removed by requiring $\log (z-t)$ to be real when $z-t>0$. In terms of gamma function,

$$
D_{z}^{-\lambda} z^{\rho}=\frac{\Gamma(\rho+1)}{\Gamma(\rho+\delta+1)} z^{\rho+\lambda}
$$

The generalized Ruscheweyh derivatives $\mathbf{J}_{p}^{\lambda, \mu} f, \mu>-1$ of $f \in A_{p}$ is defined by Goyal and Goyal [2] as follows:

$$
\begin{aligned}
& \mathbf{J}_{p}^{\lambda, \mu} f(z)=\frac{\Gamma(\mu-\lambda+\nu+2)}{\Gamma(\nu+2) \Gamma(\mu+1)} z^{p} J_{0, z}^{\lambda, \mu, \nu}\left(z^{\mu-p} f(z)\right) \\
& =z^{p}-\sum_{k=n+p}^{\infty} a_{k} B_{p}^{\lambda, \mu}(k) z^{k}
\end{aligned}
$$

where

$$
B_{p}^{\lambda, \mu}(k)=\frac{\Gamma(k-p+1+\mu) \Gamma(\nu+2+\mu-\lambda) \Gamma(k+\nu-p+2)}{\Gamma(k-p+1) \Gamma(k+\nu-p+2+\mu-\lambda) \Gamma(\nu+2) \Gamma(1+\mu)}
$$

For $\lambda=\mu$, this generalized Ruscheweyh derivatives get reduced to Ruscheweyh derivatives of $\mathrm{f}(\mathrm{z})$ of order $\lambda$ (see, e.g. [12]):

$$
\begin{aligned}
& D^{\lambda} f(z)=\frac{z^{p}}{\Gamma(\lambda+1)} \frac{d^{\lambda}}{d z^{\lambda}}\left(z^{\lambda-p} f(z)\right) \\
& =z^{p}+\sum_{k=n+p}^{\infty} a_{k} B_{k}(\lambda) z^{k}
\end{aligned}
$$


where

$$
B_{k}(\lambda)=\frac{\Gamma(\lambda+k)}{\Gamma(\lambda+p) \Gamma(k-p+1)}
$$

For $\mathrm{p}=1$, (1.11) reduces to ordinary Ruscheweyh derivatives for univalent functions [8].

The operation $*$ is the convolution (Hadamard product) of two power series

$$
f(z)=z^{p}-\sum_{k=n+p}^{\infty} a_{k} z^{k} \text { and } g(z)=z^{p}-\sum_{k=n+p}^{\infty} b_{k} z^{k}
$$

defined as

$$
(f * g)(z)=z^{p}-\sum_{k=n+p}^{\infty} a_{k} b_{k} z^{k}
$$

A function $f \in \Omega_{p}$ is said to be in the class $\Omega_{p}(\alpha, \beta, \lambda)$ if and only if

$$
\operatorname{Re}\left\{\frac{z\left(\mathbf{J}_{p}^{\lambda, \mu} f(z)\right)^{\prime}}{(1-\alpha)\left(\mathbf{J}_{p}^{\lambda, \mu} f(z)\right)+\alpha z^{2}\left(\mathbf{J}_{p}^{\lambda, \mu} f(z)\right)^{\prime \prime}}\right\}>\beta
$$

for $z \in U$ and $0 \leq \alpha<1,0 \leq \beta<p$ and $\lambda>-1$.

The class $\Omega_{p}(\alpha, \beta, \lambda)$ contains many well-known classes of analytic functions such as:

- For $\alpha=\lambda=0, \Omega_{p}(\alpha, \beta, \lambda)$ reduces to the class $S^{*}(\beta)$ of starlike functions of order $\beta$.

- For $\alpha=\lambda=-1, \Omega_{p}(\alpha, \beta, \lambda)$ reduces to the class $K(\beta)$ of convex functions of order $\beta$.

\section{Main Results}

The coefficient bounds for the functions $f \in \Omega_{p}(\alpha, \beta, \lambda)$ are found in the following theorem:

Theorem 2.1. Let $f \in A_{p}, z \in U$ be of the form (1.1). Then $f \in$ $\Omega_{p}(\alpha, \beta, \lambda)$ iff

$$
\sum_{k=n+p}^{\infty} \frac{\alpha \beta\left(1+k-k^{2}\right)+k-\beta}{p-\beta+\alpha \beta\left(1+p-p^{2}\right)} B_{p}^{\lambda, \mu}(k) a_{k}<1
$$

where $0 \leq \alpha<1,0 \leq \beta<p$ and $\lambda>-1$. 
Proof. Since $f \in \Omega_{p}(\alpha, \beta, \lambda)$

$$
\operatorname{Re}\left\{\frac{z\left(\mathbf{J}_{p}^{\lambda, \mu} f(z)\right)^{\prime}}{(1-\alpha)\left(\mathbf{J}_{p}^{\lambda, \mu} f(z)\right)+\alpha z^{2}\left(\mathbf{J}_{p}^{\lambda, \mu} f(z)\right)^{\prime \prime}}\right\}>\beta
$$

Making use of equation (1.9) in the above inequality, we obtain

$\operatorname{Re}\left\{\frac{p z^{p-1}-\sum_{k=n+p}^{\infty} k a_{k} B_{p}^{\lambda, \mu}(k) z^{k-1}}{(1-\alpha+\alpha p(p-1)) z^{p}-\sum_{k=n+p}^{\infty}[(1-\alpha)+\alpha(k(k-1))] a_{k} B_{p}^{\lambda, \mu}(k) z^{k}}\right\}>\beta$

Therefore, we obtain

$$
\sum_{k=n+p}^{\infty} \frac{\alpha \beta\left(1+k-k^{2}\right)+k-\beta}{p-\beta+\alpha \beta\left(1+p-p^{2}\right)} B_{p}^{\lambda, \mu}(k) a_{k}<1 .
$$

In this theorem, we will show that this class is closed under linear combination.

Theorem 2.2. Let for $j \in\{1,2,3, \ldots, m\}$

$$
f_{j}(z)=z^{p}-\sum_{k=n+p}^{\infty} a_{k, j} z^{k} \in \Omega_{p}(\alpha, \beta, \lambda)
$$

Then for $0<P_{j}<1, \sum_{j=1}^{m} P_{j}=1$, the function $F(z)$ defined by

$$
F(z)=\sum_{j=1}^{m} P_{j} f_{j}(z)
$$

is also in $\Omega_{p}(\alpha, \beta, \lambda)$.

Proof. For every $j \in\{1,2,3, \ldots, m\}$, we obtain

$$
\sum_{k=n+p}^{\infty} \frac{\alpha \beta\left(1+k-k^{2}\right)+k-\beta}{p-\beta+\alpha \beta\left(1+p-p^{2}\right)} B_{p}^{\lambda, \mu}(k) a_{k, j}<1 .
$$

Since

$$
F(z)=\sum_{j=1}^{m} P_{j}\left(z^{p}-\sum_{k=n+p}^{\infty} a_{k, j} z^{k}\right)=z^{p}-\sum_{k=n+p}^{\infty}\left(\sum_{j=1}^{m} P_{j} a_{k, j}\right) z^{k}
$$


Therefore,

(2.8) $\sum_{k=n+p}^{\infty} \frac{\alpha \beta\left(1+k-k^{2}\right)+k-\beta}{p-\beta+\alpha \beta\left(1+p-p^{2}\right)} B_{p}^{\lambda, \mu}(k)\left(\sum_{j=1}^{m} P_{j} a_{k, j}\right)<\sum_{j=1}^{m} P_{j}=1$

which proves the Theorem.

Theorem 2.3. Let

$$
f(z)=z^{p}-\sum_{k=n+p}^{\infty} a_{k} z^{k}
$$

and

$$
g(z)=z^{p}-\sum_{k=n+p}^{\infty} b_{k} z^{k}
$$

belong to $\Omega_{p}(\alpha, \beta, \lambda)$. Then the function

$$
G(z)=z^{p}-\sum_{k=n+p}^{\infty}\left(a_{k}^{2}+b_{k}^{2}\right) z^{k}
$$

is in $\in \Omega_{p}\left(\alpha, \beta, \lambda_{1}\right)$, where

$$
\lambda_{1} \leq \inf _{k}\left[\frac{(k-p)\left[\alpha \beta\left(1+k-k^{2}\right)+k-\beta\right]}{2\left[p-\beta+\alpha \beta\left(1+p-p^{2}\right)\right]}\left(B_{p}^{\lambda, \mu}(k)\right)^{2}-1\right] .
$$

Proof.

Since $f, g \in \Omega_{p}(\alpha, \beta, \lambda)$,

$$
\begin{gathered}
\sum_{k=n+p}^{\infty}\left[\frac{\alpha \beta\left(1+k-k^{2}\right)+k-\beta}{p-\beta+\alpha \beta\left(1+p-p^{2}\right)} B_{p}^{\lambda, \mu}(k)\right]^{2} a_{k}^{2} \\
\leq\left[\sum_{k=n+p}^{\infty} \frac{\alpha \beta\left(1+k-k^{2}\right)+k-\beta}{p-\beta+\alpha \beta\left(1+p-p^{2}\right)} B_{p}^{\lambda, \mu}(k) a_{k}\right]^{2}<1 .
\end{gathered}
$$

Similarly,

$$
\sum_{k=n+p}^{\infty}\left[\frac{\alpha \beta\left(1+k-k^{2}\right)+k-\beta}{p-\beta+\alpha \beta\left(1+p-p^{2}\right)} B_{p}^{\lambda, \mu}(k)\right]^{2} b_{k}^{2}
$$


$\leq\left[\sum_{k=n+p}^{\infty} \frac{\alpha \beta\left(1+k-k^{2}\right)+k-\beta}{p-\beta+\alpha \beta\left(1+p-p^{2}\right)} B_{p}^{\lambda, \mu}(k) b_{k}\right]^{2}<1$.

Therefore

$$
\sum_{k=n+p}^{\infty} \frac{1}{2}\left[\frac{\alpha \beta\left(1+k-k^{2}\right)+k-\beta}{p-\beta+\alpha \beta\left(1+p-p^{2}\right)} B_{p}^{\lambda, \mu}(k)\right]^{2}\left(a_{k}^{2}+b_{k}^{2}\right)<1
$$

Now, we must show that

$$
\sum_{k=n+p}^{\infty} \frac{\alpha \beta\left(1+k-k^{2}\right)+k-\beta}{p-\beta+\alpha \beta\left(1+p-p^{2}\right)} B_{p}^{\lambda_{1}, \mu}(k)\left(a_{k}^{2}+b_{k}^{2}\right)<1
$$

This inequality holds if

$$
\frac{\alpha \beta\left(1+k-k^{2}\right)+k-\beta}{p-\beta+\alpha \beta\left(1+p-p^{2}\right)} B_{p}^{\lambda_{1}, \mu}(k) \leq \frac{1}{2}\left[\frac{\alpha \beta\left(1+k-k^{2}\right)+k-\beta}{p-\beta+\alpha \beta\left(1+p-p^{2}\right)} B_{p}^{\lambda, \mu}(k)\right]^{2}
$$

which is equivalent to

$$
B_{p}^{\lambda_{1}, \mu}(k)=\frac{1}{2} \frac{\alpha \beta\left(1+k-k^{2}\right)+k-\beta}{p-\beta+\alpha \beta\left(1+p-p^{2}\right)}\left[B_{p}^{\lambda, \mu}(k)\right]^{2}
$$

Since $\frac{\lambda_{1}+1}{k-p} \leq B_{p}^{\lambda_{1}, \mu}(k)$, we obtain

$$
\frac{\lambda_{1}+1}{k-p} \leq \frac{1}{2} \frac{\alpha \beta\left(1+k-k^{2}\right)+k-\beta}{p-\beta+\alpha \beta\left(1+p-p^{2}\right)}\left[B_{p}^{\lambda, \mu}(k)\right]^{2}
$$

and this gives the required result.

A modified Komatu operator $K_{c}^{\gamma}: A \rightarrow A$ is defined for $\gamma \geq 0$ and $c>-p$ as

$$
K_{c, p}^{\gamma} f(z)=\frac{(c+p)^{\gamma}}{\Gamma(\gamma) z^{c}} \int_{0}^{1} t^{c}\left(\log \frac{1}{t}\right)^{\gamma-1} f(t z) d t
$$

It can be easily verified that for $f \in A_{p}$

$$
K_{c, p}^{\gamma} f(z)=z^{p}-\sum_{k=p+1}^{\infty}\left(\frac{c+p}{c+k}\right)^{\gamma} a_{k} z^{k}
$$


Theorem 2.4. If $f \in \Omega_{p}(\alpha, \beta, \lambda)$, then $K_{c, p}^{\gamma} f \in \Omega_{p}(\alpha, \beta, \lambda)$.

Proof. Since $f \in \Omega_{p}(\alpha, \beta, \lambda)$ and $\left\{\frac{c+p}{c+k}\right\}^{\gamma}<1$, we have

$$
\sum_{k=n+p}^{\infty} \frac{\alpha \beta\left(1+k-k^{2}\right)+k-\beta}{p-\beta+\alpha \beta\left(1+p-p^{2}\right)}\left(\frac{c+p}{c+k}\right)^{\gamma} B_{p}^{\lambda, \mu}(k) a_{k}<1
$$

This completes the proof.

\section{Radius of starlikeness and convexity}

Now we obtain the radii of starlikeness and convexity for the functions $K_{c, p}^{\gamma} f$.

Theorem 3.1. The function $K_{c, p}^{\gamma} f$ is starlike of order $\eta$ in

$$
\begin{aligned}
& |z|<r_{1}(\alpha, \beta, \lambda, c, \gamma, \eta) \text { where } \\
& \qquad r_{1}(\alpha, \beta, \lambda, c, \gamma, \eta) \\
& =\inf _{k}\left[\frac{1-\eta}{k-\eta-p+1} \frac{\alpha \beta\left(1+k-k^{2}\right)+k-\beta}{p-\beta+\alpha \beta\left(1+p-p^{2}\right)}\left(\frac{c+p}{c+k}\right)^{\gamma} B_{p}^{\lambda, \mu}(k) a_{k}\right]^{\frac{1}{k-p}}
\end{aligned}
$$

Proof. We must show that

$$
\left|\frac{z\left(K_{c, p}^{\gamma} f(z)\right)^{\prime}}{K_{c, p}^{\gamma} f(z)}-p\right|<1-\eta
$$

i.e.

$$
\left|\frac{z\left(K_{c, p}^{\gamma} f(z)\right)^{\prime}-p K_{c, p}^{\gamma} f(z)}{K_{c, p}^{\gamma} f(z)}\right| \leq \frac{\sum_{k=n+p}^{\infty}\left(\frac{c+p}{c+k}\right)^{\gamma}(k-p) a_{k}|z|^{k-p}}{1-\sum_{k=n+p}^{\infty}\left(\frac{c+p}{c+k}\right)^{\gamma} a_{k}|z|^{k-p}}<1-\eta
$$

or to show that

$$
\sum_{k=n+p}^{\infty}\left(\frac{c+p}{c+k}\right)^{\gamma}(k-p) a_{k}|z|^{k-p}+(1-\eta) \sum_{k=n+p}^{\infty}\left(\frac{c+p}{c+k}\right)^{\gamma} a_{k}|z|^{k-p}<1-\eta
$$


Now, in view of (2.1), the theorem holds if

$$
|z|^{k-p}<\frac{1-\eta}{k-p+1-\eta} \frac{\alpha \beta\left(1+k-k^{2}\right)+k-\beta}{p-\beta+\alpha \beta\left(1+p-p^{2}\right)}\left(\frac{c+p}{c+k}\right)^{\gamma} B_{p}^{\lambda, \mu}(k)
$$

This proves the result.

Theorem 3.2. The function $K_{c, p}^{\gamma} f$ is convex of order $\eta$ in

$$
|z|<r_{2}(\alpha, \beta, \lambda, c, \gamma, \eta) \text {, where }
$$

$$
\begin{gathered}
r_{2}(\alpha, \beta, \lambda, c, \gamma, \eta) \\
=\inf _{k}\left[\frac{p(p-\eta)}{k(k-\eta)} \frac{\alpha \beta\left(1+k-k^{2}\right)+k-\beta}{p-\beta+\alpha \beta\left(1+p-p^{2}\right)}\left(\frac{c+p}{c+k}\right)^{\gamma} B_{p}^{\lambda, \mu}(k) a_{k}\right]^{\frac{1}{k-p}}
\end{gathered}
$$

Proof. Noting the fact that $K_{c, p}^{\gamma} f$ is convex iff $z\left(K_{c, p}^{\gamma} f\right)^{\prime}$ is starlike. Therefore, we must show that,

$$
\left|\frac{z\left(K_{c, p}^{\gamma} f(z)\right)^{\prime \prime}}{\left(K_{c, p}^{\gamma} f(z)\right)^{\prime}}\right|<1-\eta
$$

i.e.

$$
\frac{p(p-1) z^{p-1}-\sum_{k=n+p}^{\infty}\left(\frac{c+p}{c+k}\right)^{\gamma}(k-p) a_{k}|z|^{k-1}}{p z^{p-1}-\sum_{k=n+p}^{\infty}\left(\frac{c+p}{c+k}\right)^{\gamma} a_{k}|z|^{k-1}}<1-\eta
$$

or to show that

$$
\sum_{k=n+p}^{\infty}\left(\frac{c+p}{c+k}\right)^{\gamma}[k(k-\eta)] a_{k}|z|^{k-p}<p(p-\eta)
$$

Now, by (2.1), the last inequality holds if

$$
|z|^{k-p}<\frac{p(p-\eta)}{k(k-\eta)} \frac{\alpha \beta\left(1+k-k^{2}\right)+k-\beta}{p-\beta+\alpha \beta\left(1+p-p^{2}\right)}\left(\frac{c+p}{c+k}\right)^{\gamma} B_{p}^{\lambda, \mu}(k)
$$

This complete the proof. 
Theorem 3.3. If $f \in \Omega_{p}(\alpha, \beta, \lambda)$, then the function $F_{p}^{\mu}(z), z \in U$ defined by

$$
F_{p}^{\mu}(z)=(1-\mu) z^{p}+p \mu \int_{0}^{z} \frac{f(t)}{t} d t, \quad 0 \leq \mu<\frac{2}{p}
$$

is in $\Omega_{p}(\alpha, \beta, \lambda)$.

Proof. We have

$F_{p}^{\mu}(z)=(1-\mu) z^{p}+p \mu\left[\int_{0}^{z} t^{p-1} d t-\sum_{k=n+p}^{\infty} \int_{0}^{z} a_{k} t^{k-1} d t\right]=z^{p}-\sum_{k=n+p}^{\infty} a_{k} \frac{p \mu}{k} z^{k}$

Now, by (2.1), we obtain

$$
\begin{gathered}
\sum_{k=n+p}^{\infty} \frac{\alpha \beta\left(1+k-k^{2}\right)+k-\beta}{p-\beta+\alpha \beta\left(1+p-p^{2}\right)}\left(\frac{p \mu}{k}\right) B_{p}^{\lambda, \mu}(k) a_{k} \\
\leq \sum_{k=n+p}^{\infty} \frac{\alpha \beta\left(1+k-k^{2}\right)+k-\beta}{p-\beta+\alpha \beta\left(1+p-p^{2}\right)}\left(\frac{2}{k}\right) B_{p}^{\lambda, \mu}(k) a_{k}<1
\end{gathered}
$$

and this proves the theorem.

Remark 3.4. $F_{p}^{\mu}(z)$ is starlike of order $\eta$ in $|z|<r_{1}^{p}(\alpha, \beta, \lambda, \eta, \mu)$, where

$$
\begin{gathered}
r_{1}^{p}(\alpha, \beta, \lambda, \eta, \mu) \\
=\inf _{k}\left[\frac{k(1-\eta)}{p \mu(p-k+1-\eta)} \frac{\alpha \beta\left(1+k-k^{2}\right)+k-\beta}{p-\beta+\alpha \beta\left(1+p-p^{2}\right)} B_{p}^{\lambda, \mu}(k) a_{k}\right]^{\frac{1}{k-p}} .
\end{gathered}
$$

Also, $F_{p}^{\mu}(z)$ is convex of order $\eta$ in $|z|<r_{1}^{p}(\alpha, \beta, \lambda, \eta, \mu)$, where

$$
r_{2}^{p}(\alpha, \beta, \lambda, \eta, \mu)=\inf _{k}\left[\frac{(p-\eta)}{p \mu(k-\eta)} \frac{\alpha \beta\left(1+k-k^{2}\right)+k-\beta}{p-\beta+\alpha \beta\left(1+p-p^{2}\right)} B_{p}^{\lambda, \mu}(k) a_{k}\right]^{\frac{1}{k-p}} .
$$

The proof of the above remark is made by similar arguments of the Theorems 3.1 and 3.2 . 


\section{Fractional Calculus on $\Omega_{p}(\alpha, \beta, \lambda)$}

In this section, we apply the fractional calulus techniques and discuss the properties of the family $\Omega_{p}(\alpha, \beta, \lambda)$ (see [10]). In this theorem, we find the distortion bounds for $f(z)$.

Theorem 4.1. Let $f \in \Omega_{p}(\alpha, \beta, \lambda), \lambda \geq 0$. Then

$|z|^{p+\delta} \frac{\Gamma(p+1)}{\Gamma(p+\delta+1)}\left[1-M|z|^{n}\right] \leq\left|D_{z}^{-\delta} f(z)\right| \leq|z|^{p+\delta} \frac{\Gamma(p+1)}{\Gamma(p+\delta+1)}\left[1+M|z|^{n}\right]$

where

$M=\frac{(p+1)_{n}\left[p-\beta+\alpha \beta\left(1+p-p^{2}\right)\right](\nu+\mu-\lambda+2)_{n} \Gamma(n+1)}{(p+\delta+1)_{n}\left[p+n+\beta\left\{\alpha\left(1+p+n-(p+n)^{2}\right)-1\right\}\right](\mu+1)_{n}(\nu+2)_{n}}$

and $f(z)$ is analytic function

Proof.

By equation (1.8), we have

$$
\frac{\Gamma(\delta+p+1)}{\Gamma(p+1)} z^{-\delta} D_{z}^{-\delta} f(z)=z^{p}-\sum_{k=n+p}^{\infty} a_{k} H_{p}(k, \delta) z^{k}
$$

where

$$
H_{p}(k, \delta)=\frac{\Gamma(\delta+p+1) \Gamma(k+1)}{\Gamma(k+\delta+1) \Gamma(p+1)}
$$

But $H_{p}(k, \delta)$ is a decreasing function for $k \geq n+p$ and also $B_{p}^{\lambda, \mu}(k)$ is increasing function of $k$, thus, we have

$$
H_{p}(k, \delta) \leq \frac{\Gamma(\delta+p+1) \Gamma(n+p+1)}{\Gamma(n+p+\delta+1) \Gamma(p+1)}=\frac{(p+1)_{n}}{(\delta+p+1)_{n}}
$$

and

$$
B_{p}^{\lambda, \mu}(k) \geq \frac{(\mu+1)_{n}(\nu+2)_{n}}{(\mu-\lambda+\nu+2)_{n} \Gamma(n+1)}
$$

So, we conclude that

$$
\begin{array}{r}
\left|\frac{\Gamma(\delta+p+1)}{\Gamma(p+1)} z^{-\delta} D_{z}^{-\delta} f(z)\right| \\
\leq|z|^{p}+\frac{(p+1)_{n}}{(\delta+p+1)_{n}}|z|^{n+p} \sum_{k=n+p}^{\infty} a_{k}
\end{array}
$$




$$
\leq|z|^{p} \frac{\Gamma(p+1)}{\Gamma(p+\delta+1)}\left[1+M|z|^{n+p}\right]
$$

where $\mathrm{M}$ is defined in the theorem statement. Thus, we get

$$
\left|D_{z}^{-\delta} f(z)\right| \leq|z|^{p+\delta} \frac{\Gamma(p+1)}{\Gamma(\delta+p+1)}\left[1+M|z|^{n}\right]
$$

Also, we have

$$
\begin{aligned}
& \quad\left|\frac{\Gamma(\delta+p+1)}{\Gamma(p+1)} z^{-\delta} D_{z}^{-\delta} f(z)\right| \\
& \geq|z|^{p}-\frac{(p+1)_{n}}{(\delta+p+1)_{n}}|z|^{n+p} \sum_{k=n+p}^{\infty} a_{k} \\
& \geq|z|^{p} \frac{\Gamma(p+1)}{\Gamma(p+\delta+1)}\left[1-M|z|^{n}\right]
\end{aligned}
$$

$$
\left|D_{z}^{-\delta} f(z)\right| \geq|z|^{p+\delta} \frac{\Gamma(p+1)}{\Gamma(\delta+p+1)}\left[1-M|z|^{n}\right]
$$

This completes the proof of the theorem.

Theorem 4.2. Let $f \in \Omega_{p}(\alpha, \beta, \lambda), \lambda \geq 0$. Then

$$
|z|^{p-\delta} \frac{\Gamma(p+1)}{\Gamma(p-\delta+1)}\left[1-N|z|^{n}\right] \leq\left|D_{z}^{\delta} f(z)\right| \leq|z|^{p-\delta} \frac{\Gamma(p+1)}{\Gamma(p-\delta+1)}\left[1+N|z|^{n}\right]
$$

where

$N=\frac{(p+1)_{n}\left[p-\beta+\alpha \beta\left(1+p-p^{2}\right)\right](\nu+\mu-\lambda+2)_{n} \Gamma(n+1)}{(p-\delta+1)_{n}\left[p+n+\beta\left\{\alpha\left(1+p+n-(p+n)^{2}\right)-1\right\}\right](\mu+1)_{n}(\nu+2)_{n}}$ and $f(z)$ is analytic function

Proof. By equation (1.6), we have

$$
\frac{\Gamma(p-\delta+1)}{\Gamma(p+1)} z^{\delta} D_{z}^{\delta} f(z)=z^{p}-\sum_{k=n+p}^{\infty} a_{k} R_{p}(k, \delta) z^{k}
$$


where

$$
R_{p}(k, \delta)=\frac{\Gamma(p-\delta+1) \Gamma(k+1)}{\Gamma(k-\delta+1) \Gamma(p+1)}
$$

But $R_{p}(k, \delta)$ is a decfreasing function for $k \geq n+p$ and also $B_{p}^{\lambda, \mu}(k)$ is increasing function of $k$, thus, we have

$$
R_{p}(k, \delta) \leq \frac{\Gamma(p-\delta+1) \Gamma(n+p+1)}{\Gamma(n+p-\delta+1) \Gamma(p+1)}=\frac{(p+1)_{n}}{(p-\delta+1)_{n}}
$$

and

$$
B_{p}^{\lambda, \mu}(k) \geq \frac{(\mu+1)_{n}(\nu+2)_{n}}{(\mu-\lambda+\nu+2)_{n} \Gamma(n+1)}
$$

So, we conclude that

$$
\begin{aligned}
& \quad\left|\frac{\Gamma(p-\delta+1)}{\Gamma(p+1)} z^{\delta} D_{z}^{\delta} f(z)\right| \\
& \leq|z|^{p}+\frac{(p+1)_{n}}{(p-\delta+1)_{n}}|z|^{n+p} \sum_{k=n+p}^{\infty} a_{k} \\
& \leq|z|^{p} \frac{\Gamma(p+1)}{\Gamma(p-\delta+1)}\left[1+N|z|^{n+p}\right]
\end{aligned}
$$

where $\mathrm{N}$ is defined in the theorem statement. Then, we get

$$
\left|D_{z}^{\delta} f(z)\right| \leq|z|^{p-\delta} \frac{\Gamma(p+1)}{\Gamma(p-\delta+1)}\left[1+N|z|^{n}\right]
$$

Also, we have

$$
\begin{aligned}
& \quad\left|\frac{\Gamma(p-\delta+1)}{\Gamma(p+1)} z^{\delta} D_{z}^{\delta} f(z)\right| \\
& \geq|z|^{p}-\frac{(p+1)_{n}}{(p-\delta+1)_{n}}|z|^{n+p} \sum_{k=n+p}^{\infty} a_{k} \\
& \geq|z|^{p} \frac{\Gamma(p+1)}{\Gamma(p+\delta+1)}\left[1-N|z|^{n}\right]
\end{aligned}
$$

Then

$$
\left|D_{z}^{\delta} f(z)\right| \geq|z|^{p-\delta} \frac{\Gamma(p+1)}{\Gamma(p-\delta+1)}\left[1-N|z|^{n}\right]
$$

This completes the proof.

Letting $\delta=1$ in Theorem 4.1, we obtain 
Corollary 4.3. Let $f \in \Omega_{p}(\alpha, \beta, \lambda), \lambda \geq 0$. Then

$$
\frac{|z|^{p+1}}{(p+1)}\left[1-M|z|^{n}\right] \leq\left|\int_{0}^{z} f(t) d t\right| \leq \frac{|z|^{p+1}}{(p+1)}\left[1+M|z|^{n}\right]
$$

where

$M=\frac{(p+1)_{n}\left[p-\beta+\alpha \beta\left(1+p-p^{2}\right)\right](\nu+\mu-\lambda+2)_{n} \Gamma(n+1)}{(p+n+1)_{n}\left[p+n+\beta\left\{\alpha\left(1+p+n-(p+n)^{2}\right)-1\right\}\right](\mu+1)_{n}(\nu+2)_{n}}$

and $f(z)$ is analytic function

Letting $\delta=0$ in Theorem 4.2, we obtain

Corollary 4.4. Let $f \in \Omega_{p}(\alpha, \beta, \lambda), \lambda \geq 0$. Then

$$
|z|^{p}\left[1-N|z|^{n}\right] \leq|f(z)| \leq|z|^{p}\left[1+N|z|^{n}\right]
$$

where

$$
N=\frac{\left[p-\beta+\alpha \beta\left(1+p-p^{2}\right)\right](\nu+\mu-\lambda+2)_{n} \Gamma(n+1)}{\left[p+n+\beta\left\{\alpha\left(1+p+n-(p+n)^{2}\right)-1\right\}\right](\mu+1)_{n}(\nu+2)_{n}}
$$

and $f(z)$ is analytic function

\section{References}

[1] Altinas O. and Owa S.: Neighborhoods of certain analytic functions with negative coefficients, Internet J. Math. Sci., 19, pp. 797-800, (1996).

[2] Goyal S.P. and Goyal R.: On a class of multivalent functions defined by generalized Ruscheweyh derivatives involving a general fractional derivative operator, J. Indian Acad. Math., 27 (2), pp. 439-456, (2005).

[3] Kanas K. and Wisniowska A.: Conic regions and k-uniformly convexity II, Folia Sci. Tech.Reso., 170, pp. 65-78, (1998).

[4] Komatu Y.: On analytic prolongation of a family of operators , Mathematica(cluj), 39 (55), pp. 141-145, (1990). 
[5] Ravichandran V., Sreenivasagan N. and Srivastava H. M.: Some inequalities associated with linear operator defined for a class of multivalent functions , J. Inequal. Pure Appl. Math., 4 (4), Art.70, pp. 1-7, (2003).

[6] Ruscheweyh S.: Neighborhoods of univalent functions, Proc. Amer. Math.Soc., 81(4), pp. 521-527, (1981).

[7] Shams S. and Kulkarni S. R.: Certain properties of the class of univalent functions defined by Ruscheweyh derivatives, Bull. calcutta Math. Soc., To appear (1997).

[8] Shams, S., Kulkarni, S. R. and Jahangiri, Jay M.: On a class of univalent functions defined by Ruscheweyh derivatives, Kyungpook Math. J., 43, pp. 579-585, (2003).

[9] Silverman H.:Univalent functions with negative coefficient, Proc. Amer.Math.Soc., 51, pp. 109-116, (1975).

[10] Srivastava, H.M.: Distortion inequalities for analytic and univalent functions associated with certain fractional calculus and other linear operators (In Analytic and Geometric Inequalities and Applications eds. T. M. Rassias and H. M. Srivastava), Kluwar Academic Publishers, 478, pp. 349-374, (1999).

[11] Srivastava H. M. and Owa S. (Editors): Current topics in analytic function theory, World Scientific Publishing Company Singapore, pp. 36-47, (1992).

[12] Srivastava, H. M. and Saxena, R. K.: Operators of fractional integration and their applications, Applied Mathematics and Computation, 118, pp. 1-52, (2001).

[13] Tehranchi A. and Kulkarni S. R. : Study of the class of univalent functions with negative coefficients defined by Ruscheweyh derivatives. II, J. Raj. Acad. Phy. Sci., 5(1), pp. 105-118, (2006). 


\section{Hari Singh Parihar}

Central University of Rajasthan, NH-8, Bandersindri, Kishangarh, Ajmer, India

e-mail : harisingh.p@rediffmail.com

and

Ritu Agarwal

Malaviya National Institute of Technology, Jaipur, India

e-mail : ritugoyal.1980@gmail.com 